\title{
Ge-Sb (Germanium-Antimony)
}

\section{H. Okamoto}

According to [2001Oka], the Ge-Sb phase diagram was thermodynamically analyzed by [1989Che] and [1999Sch] based on the evaluation of this system performed by [1986Ole]. In these works, the phase diagram was characterized by a simple eutectic form with a very small retrograde solubility of $\mathrm{Sb}$ in (Ge) (max about 0.03 at.\%) and no solubility of $\mathrm{Ge}$ in $(\mathrm{Sb})$.

Figure 1 shows the Ge-Sb phase diagram calculated by thermodynamic optimization by [2011Liu]. According to Fig. 1, about 6 at.\% Ge is soluble in $(\mathrm{Sb})$. This value was obtained based on the solubility data of [2009Nas]. This measurable solubility of $\mathrm{Ge}$ in (Sb), instead of no solubility [1986Ole], was reported earlier in [Hansen] and adopted in the Ge-Sb phase diagram revised by [1986Bor], where the maximum solubility was estimated to be a little smaller (approximately 4 at.\% Ge).

\section{References}

1986Bor S. Bordas, M.T. Clavaguera-Mora, B. Legendre, and $\mathrm{H}$. Chhay, Phase Diagram of the Ternary System Ge-Sb-Te. II.
The Subternary Ge-GeTe-Sb ${ }_{2} \mathrm{Te}_{3}-\mathrm{Sb}$, Thermochim. Acta, 1986, 107, p 239-265

1986Ole R.W. Olesinski and G.J. Abbaschian, The Ge-Sb (Germanium-Antimony) System, Bull. Alloy Phase Diagr., 1986, 7(3), p 219-222

1989Che P.Y. Chevalier, A Thermodynamic Evaluation of the $\mathrm{Ge}-\mathrm{In}, \mathrm{Ge}-\mathrm{Pb}, \mathrm{Ge}-\mathrm{Sb}, \mathrm{Ge}-\mathrm{Tl}$, and Ge-Zn Systems, Thermochim. Acta, 1989, 155, p 227-240

1999Sch A. Schlieper and R. Blacknik, Investigation of the Thermodynamics of Liquid Ge-In-Te and Ge-Sb-Te Alloys, Z. Metallkd., 1999, 90(7), p 475-485

2001Oka H. Okamoto, Ge-Sb (Germanium-Antimony), J. Phase Equilib.., 2001, 22(1), p 91-92

2009Nas N. Nasir, A. Grytsiv, P. Rogl, A. Saccone, and G. Giester, Phase Equilibria in Systems Ce-M-Sb $(\mathrm{M}=\mathrm{Si}, \mathrm{Ge}, \mathrm{Sn})$ and Superstructure $\mathrm{Ce}_{12} \mathrm{Ge}_{9-x} \mathrm{Sb}_{23+x}(\mathrm{x}=3.8 \pm 0.1)$, J. Solid State Chem., 2009, 182, p 645-656

2011Liu J. Liu, C. Guo, C. Li, and Z. Du, Thermodynamic Optimization of the Ge-Sb and Ge-Sb-Sn Systems, Thermochim. Acta, 2011, 520, p 38-47

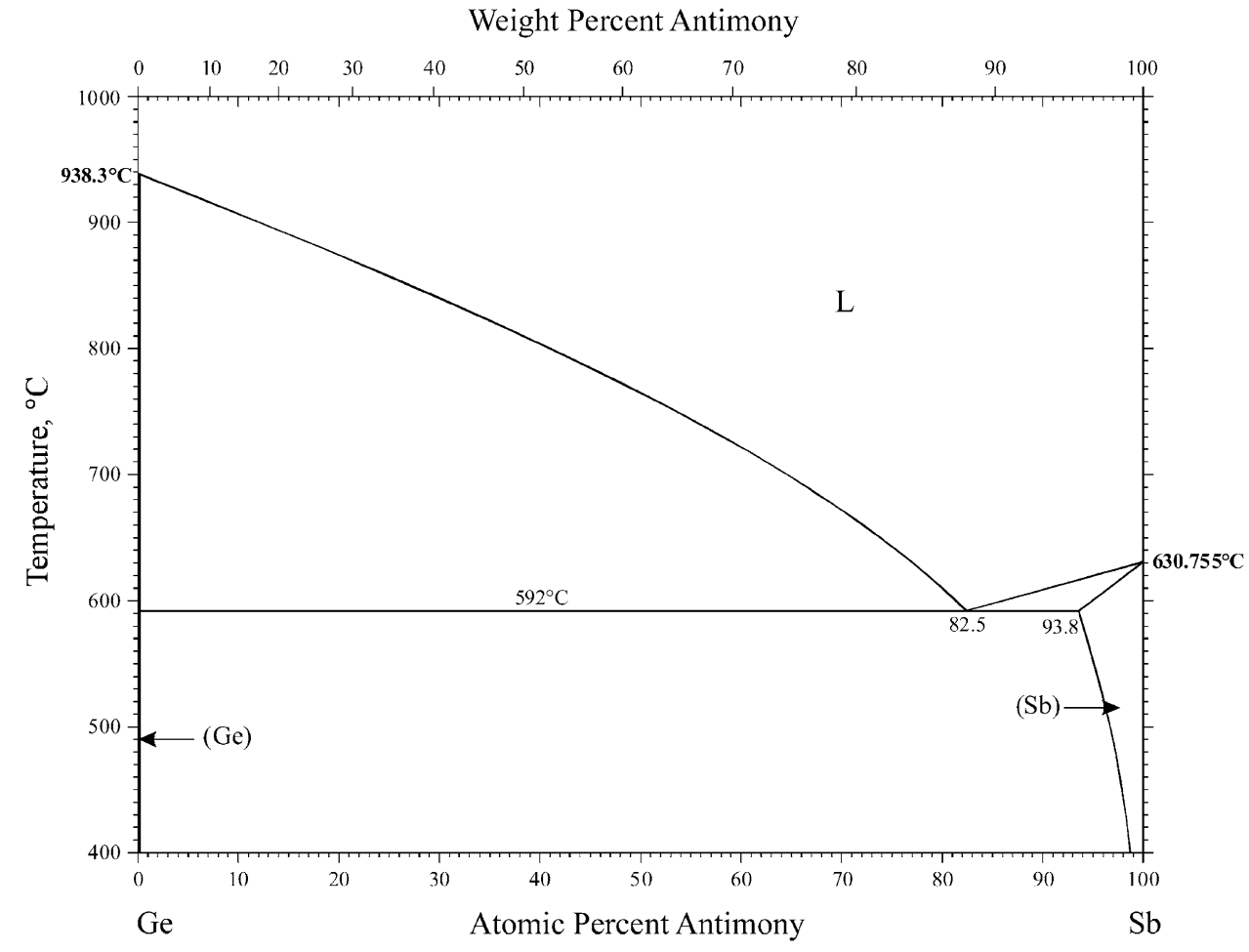

Fig. 1 Ge-Sb phase diagram 